\title{
The contribution of phonological information to visual word recognition: Evidence from Chinese Phonetic Radicals
}

Authors: Xiaodong Liu *, Luc Vermeylen, David Wisniewski, \& Marc Brysbaert

Affiliations: Department of Experimental Psychology, Ghent University, Belgium

*Corresponding author: Xiaodong Liu, Department of Experimental Psychology, Henri Dunantlaan 2, B9000, Ghent, Belgium, e-mail: xiaodong.liu@ugent.be

Declarations of interest: none 


\begin{abstract}
Lateralization is a critical characteristic of language production and also plays a role in visual word recognition. However, the neural mechanisms underlying the interactions between visual input and spoken word representations are still unclear. We investigated the contribution of sub-lexical phonological information in visual word processing by exploiting the fact that Chinese characters can contain phonetic radicals in either the left or right half of the character. FMRI data were collected while 39 Chinese participants read words in search for target color words. On the basis of whole-brain analysis and three laterality analyses of regions of interest, we argue that visual information from centrally presented Chinese characters is split in the fovea and projected to the contralateral visual cortex, from which phonological information can be extracted rapidly if the character contains a phonetic radical. Extra activation, suggestive of more effortful processing, is observed when the phonetic radical is situated in the left half of the character and therefore initially sent to the visual cortex in the right hemisphere that is less specialized for language processing. Our results are in line with the proposal that phonological information helps written word processing by means of top-down feedback.
\end{abstract}

Keywords: Chinese word processing, fMRI, assembled phonology, split fovea 


\section{Introduction}

\subsection{The network of word reading}

There is large agreement about the cortical circuits involved in word reading (see Fig. 1 for an overview, based on Dehaene, 2009). Like all visual input, written words first activate the primary visual cortex at the back of the brain. The incoming signal is further processed in higher-level areas of the visual cortex and proceeds anteriorly towards the temporal cortex, where one region responds particularly strongly to written verbal input. This ventral occipito-temporal (vOT) region has been called the Visual Word Form Area (VWFA; Cohen et al., 2002) and supports strong interactive connections with the brain areas involved in spoken word recognition and speech production, situated in temporal, parietal and frontal cortex (Fig. 1; see also Cai et al., 2008; Cai et al., 2010; Van der Haegen et al., 2012).

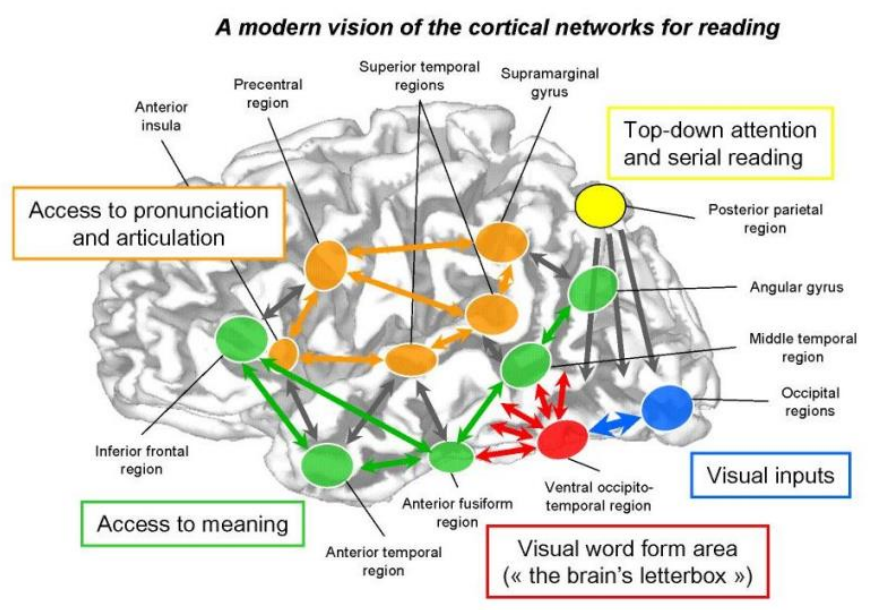

Fig. 1 Brain regions involved in word reading and their functions. Green regions are thought to be involved in access to meaning; orange regions to the interactions between a word's orthography and phonology. Source: Dehaene (2009). 
Two main routes connect the VWFA to the language-related brain areas (Carreiras et al., 2014; Taylor et al., 2013; Wandell \& Le, 2017). The first route proceeds ventrally into the middle and anterior temporal cortex and subsequently, the inferior frontal cortex. This "ventral stream" is mainly involved in the activation of word meaning and whole-word phonology (the spoken word representation). The second route proceeds dorsally and connects the VWFA with the angular gyrus and the supramarginal gyrus in parietal cortex, after which it also reaches the inferior frontal cortex. This route is called the dorsal route and is thought to predominantly mediate the sub-lexical interaction between written letters and spoken sounds. Because all connections are bidirectional, the two routes are thought to interact closely and to underlie visual word recognition together. Price and Devlin (2011) argued that the VWFA integrates the bottom-up visual input with topdown predictions generated from prior experience. As a source of top-down feedback, in particular the left inferior frontal gyrus (IFG) has been shown to modulate activity in the visual word form area in the early stages of visual word recognition (Woodhead et al., 2014).

In addition to the cortical areas traditionally investigated in visual word recognition research (summarized in Fig. 1), there is evidence for the involvement of the cerebellum (Alvarez \& Fiez, 2018), subcortical structures (Cocquyt et al., 2019) and the insula (Borowsky et al., 2006) in word reading. For instance, Alvarez and Fiez (2018) reported connectivity of the cerebellum to both the dorsal and the ventral pathway. They argued that this is because the cerebellum supports the development of fluent visual word recognition. Given that these regions were not a focus of our research, we will not discuss them further, although it may be good to keep them in mind for future research.

\subsection{Lateralization of the word reading network}


The network shown in Fig. 1 could be present in the left as well as in the right hemisphere of the brain. However, in the vast majority of people, the reading network is largely confined to the left hemisphere. Cai et al. (2008) argued that this is because speech production requires a single control center in the inferior frontal cortex. The many interactions between this region and the other language-related regions do not allow for multiple cross-hemispheric information exchanges because of the time costs involved (see also Brysbaert, 2004). As a result, the reading network largely lateralizes to the speech controlling hemisphere.

Lateralization requires transfer of information from the right hemisphere to the left hemisphere in visual word recognition, as the word part to the left of the fixation position is initially sent to the occipital cortex in the right hemisphere (Ellis \& Brysbaert, 2010; Van der Haegen et al., 2013). There are indications that part of the transfer already occurs before information reaches the visual word form area (Barca et al., 2011; Chu \& Meltzer, 2019; Selpien et al., 2015; Strother et al., 2016, 2017). In particular, Strother and colleagues presented evidence for an occipital word form area, in which considerably more transfer occurs from the right hemisphere to the left hemisphere than vice versa. The occipital word form area is situated in the inferior occipital cortex (Talairach coordinates $\mathrm{x}=-39, \mathrm{y}=-80, \mathrm{z}=-10$ ), posterior to the visual word form area (Talairach coordinates $\mathrm{x}=-40, \mathrm{y}=-59, \mathrm{z}=-10)$. A similar distinction between the middle and posterior occipito-temporal sulcus was made by Lerma-Usabiaga, Carreiras, and Paz-Alonso (2018), although their posterior part was slightly more anterior $(y=-71.5)$. Stevens et al. (2017) presented evidence that the occipital word form area may already have functional connectivity with the other language areas shown in Fig 1. Also in patients with pure alexia, there is evidence that the connections between the left occipital cortex and left vOT and IFG can be strengthened by reading training (Woodhead et al., 2013; Kerry et al., 2019). 


\subsection{The brain circuit for Chinese word recognition}

The findings discussed so far were based on alphabetic languages (mainly English), in which words are written as sequences of letters referring to the sounds of the spoken word. This is different from the logographic Chinese script, where graphic forms (characters) map onto meanings (morphemes) rather than sounds. For instance, the written word 给 (give) is pronounced as GEI in Mandarin, but as KAP in Cantonese. In addition, Chinese characters are formed with strokes in a square rather than in the linear structure of alphabetic words, and the visual complexity depends on the number of strokes in the character.

Because there are few sub-word relations between written symbols and pronunciation in Chinese (for an exception, see below), it has been suggested that the dorsal route is used differently in logographic word recognition than in alphabetic word recognition. For instance, Kawabata Duncan et al. (2014) argued that the dorsal route contributes less to the recognition of logographic Kanji words in Japanese than to the recognition of alphabetic Hiragana words. Alternatively, Tan et al. (2005) argued that the dorsal route for Chinese word reading is based on whole-word phonology (which requires the written word to be recognized before the phonology can be activated; so-called addressed phonology). This involves the middle frontal gyrus together with a phonological shortterm memory store in the inferior parietal cortex to keep the representation activated for a short period of time (Fig. 2). In contrast, alphabetic languages would make more use of letter-sound correspondences (assembled phonology), which involve the inferior frontal gyrus and a region consisting of the supramarginal gyrus in the ventral part of the inferior parietal cortex and the upper back part of the temporal gyrus. This conclusion, however, was questioned by R. Zhao, et al. (2017), who argued that the difference depends more on stimulus/task differences than language differences. 


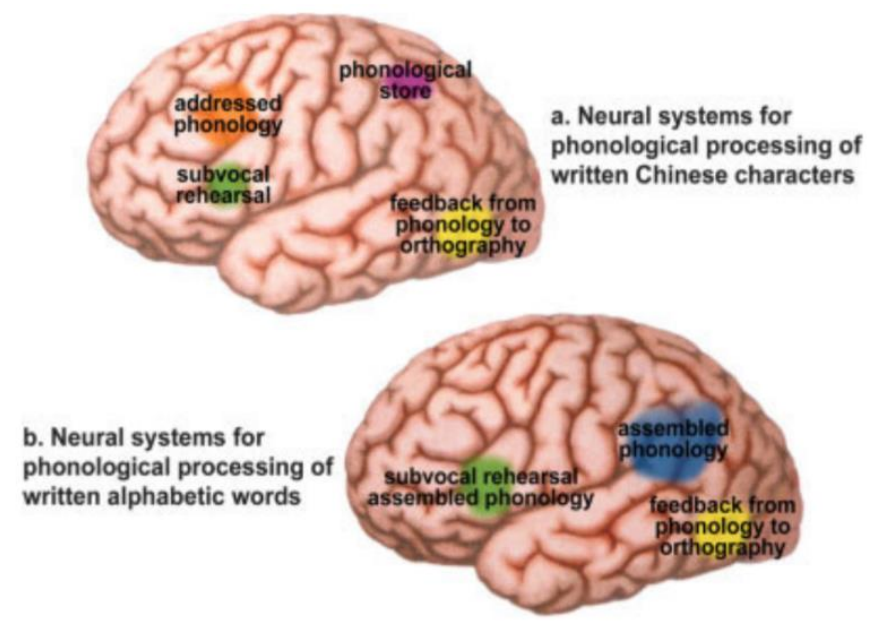

Fig. 2 Different regions assumed in the dorsal route for phonological processing of Chinese characters (a) and alphabetic words (b). Chinese word reading would mainly involve the activation of whole-word phonology (addressed phonology) in the middle frontal gyrus and short-term storage of the phonology in the inferior parietal cortex. In contrast, alphabetic word recognition would rely more on letter-to-sound correspondences (assembled phonology) involving the inferior frontal gyrus and a region involving the supramarginal gyrus in the ventral part of the inferior parietal cortex and the upper back part of the temporal gyrus. Both routes connect to the visual word form area where feedback from phonology to orthography helps in visual word recognition. Source: Tan et al. (2005)

Another difference that has been postulated between logographic and alphabetic languages is a larger involvement of the right hemisphere. For instance, it has been argued that the vOT region in the right hemisphere is more active in logographic scripts than in alphabetic scripts. Hirshorn et al. (2016) reported experimental evidence for this difference. They taught participants a new language either with an alphabetic script or with a logographic script. Greater bilateral activation was observed in the visual word form area (Talairach coordinates $\pm 45,-57,-12$ ) for the 
logographic script than for the alphabetic script. More evidence for bilateral processing in Chinese word recognition was reported in meta-analyses (Tan et al., 2005; Bolger et al., 2005; Wu et al., 2012; Zhu et al., 2014).

Evidence for bilateral processing in Chinese word recognition was also presented by L. Zhao et al. (2017). By using words that differed in orthographic and phonological similarity, they showed that the effects of phonological similarity could be observed in the VWFA and in an anterior region of the fusiform gyrus. Importantly, in this study the activation was very similar in the left and right cerebral hemisphere. This is different from the conclusion of a meta-analysis of $\mathrm{R}$. Zhao et al. (2017) who reported clear left hemisphere dominance for the visual word form area in Chinese word processing. Given that speech production in the vast majority of Chinese people is left lateralized (Mariën et al., 2004; Wang, 1996), it is reasonable to expect left dominance for Chinese visual word recognition as well, based on Cai et al. 's (2008) argument that cross-hemisphere interactions hinder performance.

\subsection{Using sub-lexical phonological information in Chinese words to increase our knowledge of the brain circuit of word reading}

In our discussion so far we have considered the logographic and alphabetic scripts as two clearly separated categories. This does not correspond completely to reality. In alphabetic languages the correspondence between the written letters (orthography) and word pronunciation (phonology) can be more or less transparent. Everybody with some knowledge of English pronounces the word "kiss" correctly. However, few people pronounce the word "awry" correctly without having learned the pronunciation explicitly. In irregular words such as "awry", assembled (letter-to-sound) phonology is of little use and readers have to rely on addressed phonology. 
Similarly, in a logographic script like Chinese it is not true that written characters never include sub-word cues about the pronunciation. Over $80 \%$ of the 7000 most frequent characters contain phonetic radicals helping with the pronunciation (Li \& Kang, 1993). The dominant type of Chinese characters consists of phonetic compounds, which have a phonetic radical and a semantic radical conveying information about character meaning.

A further interesting aspect of Chinese orthography is that the phonetic radical can be situated in the left or the right half of the character. According to an analysis by Hsiao and Shillcock (2006), more than $70 \%$ of phonetic compounds have a left-right configuration. Most of these have the phonetic radical to the right and the semantic radical to the left (sP characters); the remaining have the opposite arrangement (Ps characters). The ratio of sP characters to Ps characters is about 9 to 1.

The lateral position of the phonetic radical is interesting, because it increases our chances of picking up the brain signal. Given that a phonetic radical in the left half of a Chinese character is sent to the right occipital cortex and a phonetic radical in the right half of a Chinese character to the left occipital cortex (Ellis \& Brysbaert, 2010; Van der Haegen et al., 2013), chances are high that we will be able to pick up the brain signal involved in the processing of the phonetic radical by comparing activity in the left and the right brain half. Hsiao and colleagues (Hsiao et al., 2007; Hsiao \& Liu, 2010) presented some evidence for this. In an EEG study, they reported that Chinese characters with the phonetic radical to the right elicited left-lateralized processing in an early ERP component (N1, around 180-200 ms post-stimulus), whereas no lateralized processing was observed for characters with the phonetic radical to the left. Source analysis suggested that the difference in $\mathrm{N} 1$ was located close to vOT. 
More specifically, we may expect to see different brain activity due to the phonetic radical position in vOT, inferior parietal cortex, supramarginal gyrus, and/or in inferior/middle frontal cortex (Fig. 2).

To make sure that the effects are due to phonology and not to orthographic differences between the Chinese characters with phonetic radicals to the left or to the right, we made use of an additional set of Chinese character pairs that also have a left-right configuration but do not include phonetic radicals providing a clue about the pronunciation of the word. Such words are referred to as ideographs. For example, the ideograph character '休’ (xiū, meaning rest), shows a person (人, rén) resting by a tree (木, mù) and does not include a phonetic radical. Such words provide an opportunity to control for the orthographic information in the radicals, as they have the same leftright structure but do not have sub-word clues about their pronunciation.

\section{Method}

\subsection{Participants}

Forty healthy graduate students (17 males; mean age: 26.85 years; range: 23-34 years) were paid $€ 25$ to take part in the experiment. All participants were native literate Mandarin speakers with normal or corrected-to-normal vision, were right-handed according to the Edinburgh Handedness Inventory (Oldfield, 1971), and had no history of neurologic or psychiatric problems. All participants were studying at Ghent University and screened to meet all conditions for scanning. Before the experiment, participants signed an informed consent form (which was approved by the Ethics Committee of Ghent University Hospital). One participant had to be excluded because of excessive head movements during scanning (up to $5 \mathrm{~mm}$ ).

\subsection{Materials}


Three types of middle to high-frequency characters with left-right configuration were selected including two types of phonetic compounds and one control type of ideographs (Table 1). The phonetic compounds had a phonetic radical providing sub-word information about the pronunciation. Because phonetic radicals are often stand-alone characters as well, the pronunciation of a phonetic compound and its phonetic radical can differ to some extent. When a phonetic compound is pronounced the same as its phonetic radical, the character is called a regular character. If the phonetic compound and its phonetic radical are pronounced differently only in tone, the character is defined as a semi-regular character (in the rest of the article both regular and semi-regular characters are referred to as regular characters, as otherwise the number of characters that could be found was too small). Finally, when a phonetic compound and the embedded phonetic radical have different pronunciations, the character is defined as an irregular character (Hue, 1992). A further distinction can be made between irregular characters that share the onset, the rime or nothing with the embedded phonetic radical. They are called respectively alliterating, rhyming, and radically irregular characters (Hsiao \& Shillcock, 2006). Because radically irregular characters do not contain reliable sub-lexical information about word pronunciation, we did not include them in our study. So, we had two types of phonetic compounds: regular characters (RegW) and alliterating/rhyming characters (RhyW), which together make the phonetic stimuli (PhoW).

The third type of stimuli (the ideographs) were used as control stimuli, to make sure that the effects of phonetic radicals were due to phonology and not to the orthographic structure of the stimuli. These stimuli did not contain phonetic radicals but had a similar structure with sub-word information that could be situated in the left or the right half of the character. The critical radical in these words conveyed semantic information. 
In addition to the three types of stimuli, we selected stimuli according to the position of the critical radical. Half of the characters had the radical to the right ( $\mathrm{sP}$ and $\mathrm{sS}$ ), half had the radical to the left (Ps and Ss words). To make sure that any effect was due to position, the critical radicals were the same in matched sP-Ps and sS-Ss pairs. Also the pronunciation of the matched compounds characters was the same (apart from the tone), and they were similar in terms of word frequency and number of strokes. An example of each pair type is shown in Table 1. The full list of stimuli with their characteristics can be found in Appendix A. Because there are not many semantic control words in the Chinese language, we were unable to find the same number of control stimuli as phonetic stimuli taking all constraints into account (see Table 1).

Table 1. Exemplar illustration of the six types of target characters

\begin{tabular}{|c|c|c|c|c|c|c|c|c|}
\hline \multirow{2}{*}{ Type } & \multicolumn{5}{|c|}{ Example Character } & \multicolumn{2}{|c|}{ Critical Radical } & \multirow{2}{*}{ Nwords } \\
\hline & character & conf. & pinyin & frequency & Nstrokes & radical & pinyin & \\
\hline \multirow{2}{*}{ RegW } & 证 & $\mathrm{sP}$ & zheng4 & 5.78 & 7 & \multirow{2}{*}{ 正 } & \multirow{2}{*}{ zheng4 } & 42 \\
\hline & 政 & Ps & zheng4 & 6.14 & 9 & & & 42 \\
\hline \multirow{2}{*}{ RhyW } & 徐 & $\mathrm{sP}$ & $\mathrm{xu} 2$ & 4.97 & 10 & \multirow{2}{*}{ 余 } & \multirow{2}{*}{ yu2 } & 36 \\
\hline & 叙 & Ps & xu4 & 4.78 & 9 & & & 36 \\
\hline \multirow{2}{*}{ SemW } & 奢 & sS & du3 & 4.54 & 12 & \multirow{2}{*}{ 者 } & \multirow{2}{*}{ zhe3 } & 24 \\
\hline & 都 & Ss & du1 & 6.36 & 10 & & & 24 \\
\hline
\end{tabular}

Note: for each type of characters, there were no significant differences in terms of character frequency and stroke number between the matched pairs (paired t-test, n.s.). RegW are words with a phonetic radical that is pronounced the same. RhyW are words with a rhyming/alliterating phonetic radical. Together they are the phonetic stimuli (PhoW). SemW are ideographs that contain a semantic radical. Conf. $=$ configuration, $\mathbf{p}=$ phonetic radical, $s=$ semantic radical, pinyin $(1-4)=$ the tone for the pinyin pronunciation, 
frequency $=$ word frequency expressed as Zipf scores (Van Heuven et al., 2014) based on Jun (2010), Nstrokes $=$ number of strokes, Nwords $=$ number of words.

Finally, we selected 30 filler words (5 characters indicating color and 25 irrelevant characters), which we used as targets for a color search task. A group of 10 additional participants was recruited to make sure that none of our experimental stimuli were related to color. A 5- point scale was used, with $1=$ "Completely unrelated to color" to $5=$ "Completely related to color". The score for the experimental characters was $1.3(\mathrm{SD}=0.26)$, against $4.2(\mathrm{SD}=0.81)$ for the five characters indicating color. The filler stimuli were high-frequency left-right configuration characters as well. Even though these materials were not analyzed, they also belonged to one of the stimulus types described in Table 1(see Appendix A for more details).

\section{3. fMRI Procedure}

To increase power, a short block design was used for the scan. Each block contained 6 words of the same type (e.g., sP regular words) and lasted for 6s. A word started with a black-color central fixation cross against a gray background for $200 \mathrm{~ms}$, followed by the word (subtending $2^{\circ} \times 2^{\circ}$ ) for $800 \mathrm{~ms}$. The blocks were presented in a run, lasting $489.5 \mathrm{~s}$ in total, and starting and ending with a $10 \mathrm{~s}$ screen with an asterisk presented in the center. Between blocks there was a blank screen for a variable time between $3.5 \mathrm{~s}$ and $7.5 \mathrm{~s}$. In addition to the 34 critical blocks, there were 5 filler blocks that contained a color word and five unrelated fillers. The task of the participants was to read the sequence of words and press on a button whenever they saw a color word. After the participants pressed the button, the font of the presented character changed from NSimSun to NSimSun bold, so that the participants knew they had pressed the button well. In order to increase the number of observations, participants completed two runs with a break in-between. To reduce repetition priming, the target blocks and 5 filler blocks were pseudorandomized per run, with the 
restriction that no two consecutive blocks belonged to the same type of stimulus. To minimize order effects, we used 5 different pseudorandom stimuli sequences, in which the blocks and the order of stimuli within blocks (both target and filler) were different.

Duration of the stimulus words was set at $800 \mathrm{~ms}$, so that participants had time to process the meaning of the words and were not hurried to respond as rapidly as possible. This could be done because the dense nature of Chinese characters does not elicit many eye movements while reading words (which could be a problem with long alphabetic words). In order to grasp the attention of the participants, we used the color recognition task. When participants recognized a color word, they pressed a button. Both the participant in the scanner and the experimenter outside could see the change of character font. Such a meaning search task was better than reading aloud, because it reduced the risk of head movements during scanning. It was also better than tasks with non-words, because it required participants to process the meaning of the words and no manual response was required on the experimental trials. The meaning search task is known to involve the same word recognition processes as silent reading, given that the pattern of eye movements is very similar in both tasks (Alan et al., 2002; Schroyens et al., 1999).

\section{4. fMRI Data Acquisition}

Whole-brain imaging data was acquired with a 3T Magnetom Trio MRI Scanner (Siemens Medical Systems, Erlangen, Germany) with a 64-channel radiofrequency head coil at Ghent University Hospital. Stimuli were projected onto a translucent screen and perceived by participants via a mirror mounted on the head coil in front of their eyes. PsychoPy2 software (Peirce et al., 2019) was used to present the stimuli. Functional images were recorded by the multiband-accelerated Echo Planar Imaging $(\mathrm{EPI})$ sequence $(\mathrm{TR}=1000 \mathrm{~ms}, \mathrm{TE}=31 \mathrm{~ms}$, field of view $(\mathrm{FOV})=210 \mathrm{~mm}$, flip angle $=52^{\circ}$, slice thickness $=2.5 \mathrm{~mm}$, distance factor $=0 \%$, voxel size $=2.5 \times 2.5 \times 2.5 \mathrm{~mm}^{3}$, 
56 slices, multiband factor $=4)$. T1-weighted high-resolution structural images were acquired using the magnetization-prepared rapid acquisition gradient echo (MP-RAGE) sequence $(\mathrm{TR}=$ $2250 \mathrm{~ms}, \mathrm{TE}=4.18 \mathrm{~ms}$, field of view $(\mathrm{FOV})=256 \mathrm{~mm}$, flip angle $=9^{\circ}$, slice thickness $=1 \mathrm{~mm}$, distance factor $=50 \%$, voxel size $=1 \times 1 \times 1 \mathrm{~mm}^{3}, 176$ slices $)$.

\section{5. fMRI Data Analysis}

\subsubsection{Data pre-processing}

fMRI data analysis was performed with (https://www.fil.ion.ucl.ac.uk/spm/software/spm12/) implemented in Matlab2016b (MathWorks, Massachusetts, USA). During the pre-processing the field map was used to reduce spatial distortion of functional images. These functional images were then corrected for head motion and slice timing, co-registered to the participant's T1 image. Functional images were segmented and normalized to the Montreal Neurological Institute (MNI) T1 template and spatially smoothed by using a Gaussian kernel with a full width at half maximum (FWHM) $6 \mathrm{~mm}$.

\subsubsection{Statistical analyses}

The pre-processed data from each participant was entered into first-level statistical analysis. For each participant, the General Linear Model (GLM) was used to estimate the condition effects, where each experimental condition (Table 1) within per run was convolved with a canonical hemodynamic response function (HRF; Friston et al., 1994) modeled with a boxcar function. More specifically, all blocks were modeled by the boxcar function, the fixation condition was modeled implicitly, and six estimated head movement parameters were added into the models as regressorsof-no-interests. Additionally, GLM scaled the BOLD signal by the average global signal. About the contrasts of interest, we compared the two subsets of characters for each type of stimuli. These 
subsets share the same pronunciation and the same phonetic or semantic radical, but the position of the shared phonetic or sematic radical is different. So, for RegW we compared the sP arrangement against Ps to explore the regions related to RegW words with phonetic radicals on the right. In addition, we compared Ps against sP to find the regions specifically related to RegW words with phonetic radicals on the left. The same procedure was used for the RhyW and SemW stimuli. Furthermore, since the phonetic radicals embedded in both RegW and RhyW contain clues to the pronunciation of the words, we combined these two types of words as a general condition regardless of the regularity of pronunciation (PhoW). Effects were assessed for the whole-brain by adopting a random effects model. We ran one-sample t-tests for multiple testing for all interesting contrasts across all participants at group-level.

\subsubsection{Regions of interest}

Individual laterality indices were calculated for seven regions of interest (ROIs) mentioned in the Introduction: the visual word form area (VWFA), the posterior fusiform gyrus (posFG), the anterior fusiform gyrus (antFG), the inferior parietal region (IPL), the supramarginal gyrus (SG), the middle frontal gyrus (MFG) and the inferior frontal gyrus (IFG). The location of these predefined ROIs can be found in Fig. 3. The ROIs were defined before the analysis, based on the existing literature, so that we did not run the risk of overfitting the data because of too many degrees of freedom during the analysis.

All ROIs were defined in MNI standard space. We started with the left hemisphere. For the VWFA, we followed L. Zhao et al. (2017) and defined VWFA as a box ranging from $\mathrm{X}=-50$ to $-30, \mathrm{Y}=$ -70 to -50 and $Z=-18$ to -6 . L. Zhao et al. (2017) reported that this region in the middle of the fusiform gyrus includes the peak activation locations of individual participants reading Chinese words. The mask is also in line with the VWFA masks used in research with alphabetic languages 
(e.g., Twomey et al., 2011; Cai et al., 2010; Jobard et al. 2003). For instance, Twomey et al. (2011) used a mask ranging from $X=-54$ to -30 , from $Y=-70$ to -45 , and from $Z=-30$ to -4 .

Two more ROIs were defined in the vicinity of VWFA. The first mask was a region posterior to VWFA, which we call posFG. It ranged from $\mathrm{X}=-50$ to $-30, \mathrm{Y}=-90$ to -70 , and $\mathrm{Z}=-18$ to -6 . It includes the occipital word form area identified by Strother et al. $(2016,2017)$ and the posterior part of the occipito-temporal sulcus distinguished by Lerma-Usabiaga et al. (2018). The second mask, which we call antFG, was a box situated anterior to VWFA ranging from $\mathrm{X}=-50$ to $-30, \mathrm{Y}$ $=-50$ to -30 and $Z=-18$ to -6 . L. Zhao et al. (2017) identified it as another region in the fusiform gyrus important for processing Chinese words. The three predefined masks discussed so far have the same range of $\mathrm{X}$ and $\mathrm{Z}$ coordinates and occupy the entire fusiform gyrus with the $\mathrm{y}$ coordinate ranging from -30 to -90 .

The last four ROIs were cortical regions generally known to be involved in phonological word processing (Fig. 1): inferior parietal region (IPL), supramarginal gyrus (SG), middle frontal gyrus (MFG), and inferior frontal gyrus (IFG). The masks for IPL, SG, MFG were defined according to the meta-analysis of Tan et al. (2005), which focused on phonological processing in Chinese words. Tan et al. (2005) provided peak coordinates in Talairach space, which we converted to MNI space (http://sprout022.sprout.yale.edu/mni2tal/mni2tal.html). Each ROI was defined as a sphere with a radius of $8 \mathrm{~mm}$ centered at the peak. Peak coordinates for the left IPL were $(-36,-45,52)$, for SG $(-59,-30,12)$, and for MFG $(-48,24,25)$. Finally, the mask for IFG was identified as the pars opercularis (Brodmann area 44) and pars triangularis (Brodmann area 45) in the AAL template (Tzourio-Mazoyer et al., 2002).

After the ROIs in the left hemisphere were defined, we selected the ROIs in the right hemisphere as the mirror-reversed masks. 


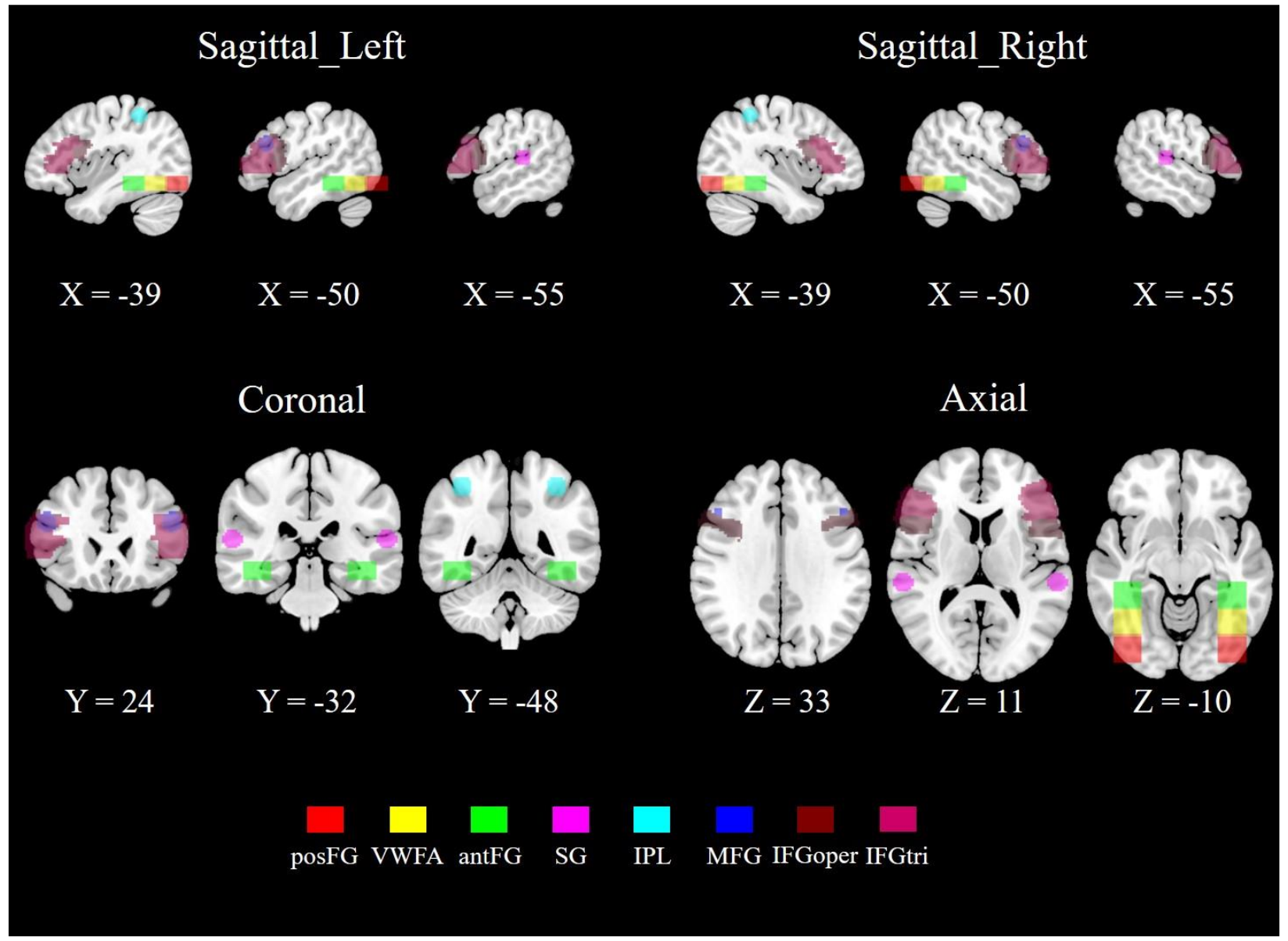

Fig. 3. The anatomical masks used on the basis of the existing literature.

\subsubsection{Laterality Indices}

Because there was no a priori guidance on the optimal analysis strategy for the functional data, we decided to run four analyses that have been proposed for laterality research, so that we could see how well the information converged. The first analysis was a whole brain analysis, to make sure we did not miss essential information in the ROI analyses. The three other analyses looked at different laterality indices (LI) in the predefined ROIs. 
Effect-size-based measure. In the first LI analysis we followed Mahowald and Fedorenko (2016), who argued that an effect-size-based measure is more reliable within subjects than a traditional volume-based measure to determine the degree of lateralization in the language system. In a traditional volume-based analysis, the degree of lateralization is measured by looking at the difference in number of activated voxels between the left hemisphere and the right hemisphere in a pre-specified ROI. However, in an effect-size-based analysis, a functional ROI (fROI) is defined within the predefined ROI and a Group-constrained Subject-Specific (GSS) analysis, created by Fedorenko et al. (2010), is used to calculate laterality. Briefly, in the GSS analysis a group-level probabilistic map of activations is used to divide the activation landscape into 'parcels'. The grouplevel parcels are used to constrain the selection of individual-level fROIs. The latter is done by selecting for each participant the voxels in the parcels with the top $10 \%$ or $20 \%$ activation. To ensure independence of data (Kriegeskorte et al., 2009), the activation levels are calculated separately from the data used to determine the fROI. The activation maps of all but one run are used to define the fROI, and the response of the remaining run is used to calculate the functional contrast of interest. The cross-validation is repeated until the entire dataset is sampled. Specific to our data, we did not have a localizer contrast but we had two runs for each subject. Therefore, we used the responses in the 7 predefined ROIs in the first run to define the fROI (based on the voxels with the top $10 \% \mathrm{t}$-values in the activation map) and we used the activation level of the second run within this fROI to determine the functional laterality index. To make sure that the effect was not limited to the second run, we repeated the procedure with a role reversal of the first and second run, and we averaged the two responses as a single response magnitude for each contrast of interest within a given predefined ROI and participant. To calculate the effect size of each contrast of interest, the percent BOLD signal change (PSC) values of each condition associated with the 
contrast were extracted from each fROI, which reflects the strength of the BOLD responses to each condition (compared to the fixation condition). We computed the effect size for the left and the right hemisphere separately and the individual LIs were calculated for each contrast of interest and

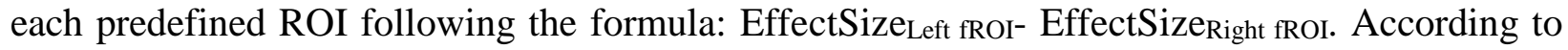
this equation, positive values $=$ left lateralization and negative values $=$ right lateralization .

LI Toolbox. The second LI analysis we ran was based on the predefined ROIs themselves rather than on a subset of most active voxels. These laterality indices were calculated with the LI toolbox of Wilke and Lidzba (2007). Wilke and Lidzba developed this toolbox to solve two long-standing problems with the lateralization index: severe threshold dependency and statistical outliers. To do so, 20 thresholds are chosen at equal intervals from 0 to the maximum t-value in the ROI. Under each threshold, 100 bootstrapped samples (sample ratio $k=0.25$ ) are taken from each side then are iteratively calculated to generate 10,000 possible LIs combinations based on the formula $[(\mathrm{L}-$ $\mathrm{R}) /(\mathrm{L}+\mathrm{R})]$. Of note, when starting the iteration (threshold=0), if the number of voxels on either of sides in the masked image is below a required minimum (default $=20$ ), the toolbox will abort the iteration. To exclude statistical outliers, the upper and the lower $25 \%$ of the LIs are excluded to keep the central $50 \%$ of data. Furthermore, a weighted mean is used to compute a mean of all remaining LIs assigned with the corresponding threshold (Wilke and Schmithorst (2006) provide more details). The LI values range from -1 to +1 . Similar to the effect-size-based measure, positive values $=$ left lateralization and negative values $=$ right lateralization.

Voxel-based laterality maps. The final LI analysis we ran was based on direct interhemispheric comparisons of signal magnitude, as proposed by Seghier and Price (2011). To do so, at the first level of analysis a symmetrical prior was used. During the data preprocessing and at the normalization-segmentation step, the default $\mathrm{T} 1$ template was replaced by a symmetrical version, 
which was created by averaging the original T1 template of SPM12 and its mirror-reversed image along the $\mathrm{x}$-axis. Functional images were segmented and normalized to this symmetrical prior, and the parameters of the normalization-segmentation processing were applied to the contrasts of interest. At the first level of analysis, voxel-based laterality maps for each participant were constructed by subtracting the left-right flipped version from the original symmetrical contrast images. This laterality map reflects the hemispheric difference for each contrast at voxel level. For each contrast, at the group level, each ROI was reported at a threshold of voxel-level, $p<0.05$ group-wise, FWE-corrected.

\section{Results}

\subsection{Behavioral Results}

No participant reported any difficulty in completing the color word searching task and participants made on average $0.6 \%$ errors. Importantly, the number of button presses in the critical blocks was less than once per participant. The few blocks with button presses were omitted from the fMRI analyses.

\section{2. fMRI Whole-brain Analysis}

First, we ran a whole-brain analysis in which we compared the words with critical information in the right half to the words with critical information in the left half for each type of words (Table 2). This analysis showed that there were more clusters surviving when the phonetic radical was situated in the left half (i.e., sent to the right hemisphere) and when the semantic control radical was situated in the right half (i.e., sent to the left hemisphere).

Table 2, Location and coordinates of peak activation for each contrast (voxel-wise threshold $P<0.001$, uncorrected, cluster size $\geq 60$ voxels). 


\begin{tabular}{|c|c|c|c|c|c|c|}
\hline \multirow{2}{*}{ Region } & \multirow{2}{*}{ Side } & \multicolumn{3}{|c|}{ MNI coordinates } & \multirow{2}{*}{$\begin{array}{l}\text { Peak } \\
\mathrm{T}\end{array}$} & \multirow{2}{*}{$\begin{array}{l}\text { Cluster } \\
\text { size }\end{array}$} \\
\hline & & $\mathrm{X}$ & $\mathrm{Y}$ & $\mathrm{Z}$ & & \\
\hline \multicolumn{7}{|l|}{ PhoW Right > Left } \\
\hline Fusiform gyrus & $\mathrm{L}$ & -40 & -58 & -16 & 4.48 & 76 \\
\hline \multicolumn{7}{|l|}{ PhoW Left > Right } \\
\hline Inferior occipital gyrus & $\mathrm{R}$ & 40 & -80 & -12 & 5.61 & 166 \\
\hline Precuneus & $\mathrm{R} / \mathrm{L}$ & 4 & -68 & 38 & 5.57 & 1663 \\
\hline Inferior parietal lobule & $\mathrm{R}$ & 46 & -66 & 38 & 5.04 & 396 \\
\hline Occipital lobe & $\mathrm{R}$ & 20 & -94 & 8 & 4.65 & 74 \\
\hline Angular gyrus & $\mathrm{L}$ & -42 & -68 & 38 & 4.89 & 376 \\
\hline Middle frontal gyrus & $\mathrm{L}$ & -20 & 10 & 48 & 4.44 & 263 \\
\hline \multicolumn{7}{|l|}{ RegW Right $>$ Left } \\
\hline Inferior occipital gyrus & $\mathrm{L}$ & -36 & -86 & -10 & 4.33 & 86 \\
\hline \multicolumn{7}{|l|}{ RegW Left $>$ Right } \\
\hline \multicolumn{7}{|l|}{ None } \\
\hline \multicolumn{7}{|l|}{ RhyW Right > Left } \\
\hline \multicolumn{7}{|l|}{ None } \\
\hline \multicolumn{7}{|l|}{ RhyW Left > Right } \\
\hline Inferior occipital gyrus & $\mathrm{R}$ & 38 & -78 & -10 & 5.33 & 355 \\
\hline Precuneus & $\mathrm{R}$ & 12 & -64 & 28 & 4.95 & 698 \\
\hline Occipital lobe & $\mathrm{R}$ & 20 & -94 & 6 & 4.89 & 61 \\
\hline Middle temporal gyrus & $\mathrm{R}$ & 42 & -64 & 28 & 4.56 & 178 \\
\hline Precuneus & $\mathrm{L}$ & -8 & -44 & 18 & 4.59 & 217 \\
\hline Angular gyrus & $\mathrm{L}$ & -40 & -68 & 36 & 4.52 & 143 \\
\hline Precentral gyrus & $\mathrm{L}$ & -34 & -22 & 60 & 3.98 & 67 \\
\hline \multicolumn{7}{|l|}{ SemW Right > Left } \\
\hline Inferior parietal lobule & $\mathrm{L}$ & -38 & -56 & 36 & 5.01 & 423 \\
\hline $\begin{array}{l}\text { Inferior frontal gyrus, } \\
\text { triangular part }\end{array}$ & $\mathrm{L}$ & -46 & 28 & 12 & 4.97 & 437 \\
\hline Inferior frontal gyrus & $\mathrm{L}$ & -30 & 28 & -12 & 4.73 & 89 \\
\hline Frontal lobe & $\mathrm{L}$ & -28 & 6 & 38 & 4.71 & 68 \\
\hline Middle frontal gyrus & $\mathrm{L}$ & -34 & 38 & -2 & 4.58 & 66 \\
\hline Fusiform gyrus & $\mathrm{L}$ & -26 & -64 & -10 & 4.22 & 75 \\
\hline Occipital lobe & $\mathrm{L}$ & 0 & -66 & 2 & 3.88 & 106 \\
\hline $\begin{array}{l}\text { SemW Left }>\text { Right } \\
\text { None }\end{array}$ & & & & & & \\
\hline
\end{tabular}


Note: Right >Left, contrast the activation of characters with the shared radical on the right to the left; Left > Right, contrast the activation for characters with the shared radical on the left to the right.

The group-level activation maps for the different word types are shown in Fig. 4 (visualized with BrainNet Viewer, Xia et al., 2013). For the words with phonetic radicals (PhoW), including all stimuli with the phonetic radical, the site of peak activation to the characters with the phonetic radical on the right was located in the left fusiform gyrus, while the characters with the phonetic radicals on the left evoked a wider range of activation, including the inferior occipital cortex, the parietal lobe, and the middle frontal gyrus bilaterally. The latter was particularly true for the RhyW stimuli. For these words, when the phonetic radical was situated on the left, the characters elicited strong activation bilaterally in the inferior occipital cortex, the middle temporal cortex, the angular gyrus and the precentral gyrus, but no regions were extra active for characters with the phonetic radical to the right. For $\mathrm{RegW}$ stimuli there was only significantly increased activation in the left VWFA when the characters had the phonetic information on the right. The laterality pattern was completely different for SemW stimuli, where extra activation was observed in the left fusiform gyrus, inferior parietal lobule and inferior/middle frontal gyrus when the critical radical was in the right half of the character. 

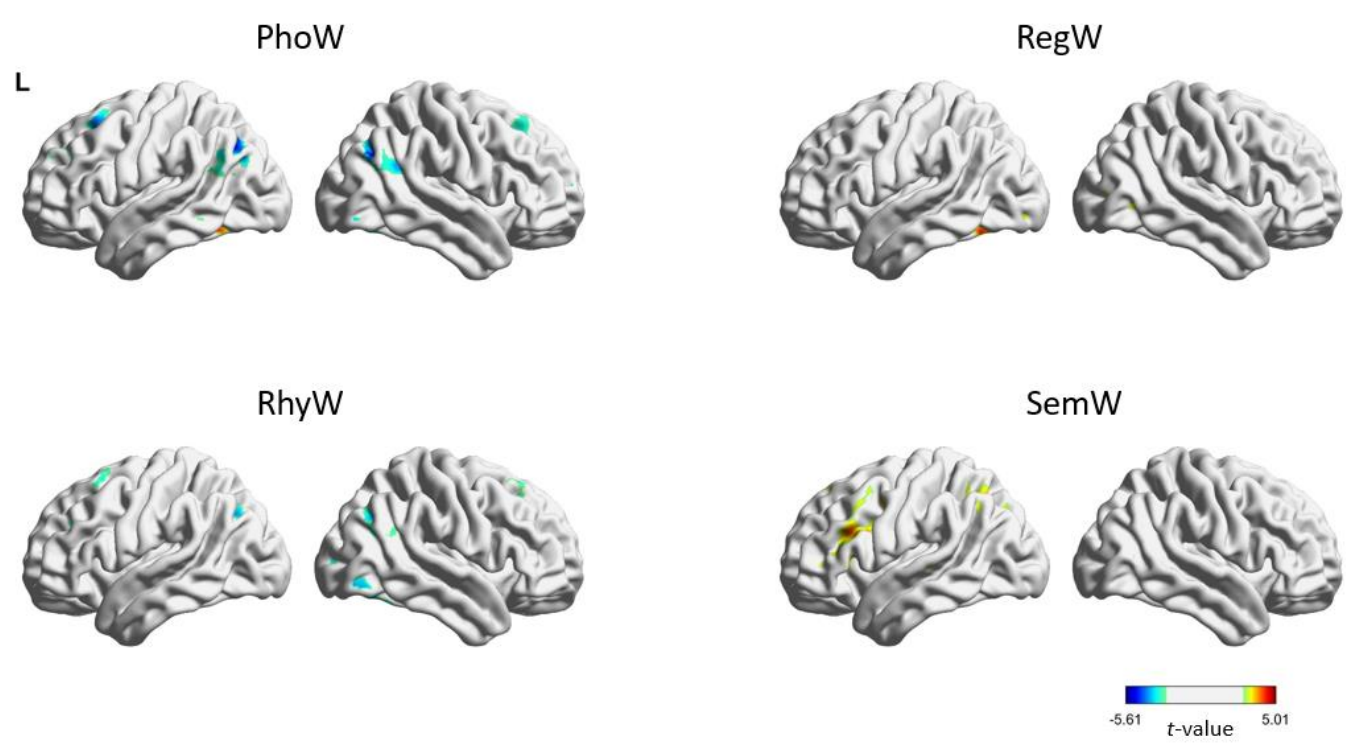

Fig. 4. Group-level brain activation for the various types of words at the whole brain. For each type of stimuli, the regions related to the characters where the shared radical was situated on the right were compared to the characters with the shared radical on the left. Stronger activation to the character with shared radicals on the right are indicated by yellow-red, while the regions related to the character with the shared radical on the left are marked by green-blue. $L=$ the left hemisphere.

\subsection{ROI Analysis}

As indicated in the method section, individual LIs were computed with three methods in predefined ROIs. First, LIs were calculated with the effect size measure (Mahowald \& Fedorenko, 2016). When the top $10 \%$ of voxels were selected as fROI, the effects were situated in posFG and VWFA (Fig. 5, one-sample t-tests, Bonferroni correction for the 7 regions). Within posFG, the two subsets of PhoW words showed totally different lateralized pattern. When the phonetic radical was situated to the left, activation was significantly larger in the right hemisphere than in the left hemisphere $\left(\right.$ Mean $\left._{\mathrm{LI}}=-0.14, \mathrm{t}(38)=-5.59, p=0.0001\right)$; when the phonetic radical was situated to the right, 
activation was significantly larger in the left hemisphere $\left(\right.$ Mean $\left._{\mathrm{LI}}=0.10, \mathrm{t}(38)=4.58, p=0.0028\right)$. The effect was stronger for words with rhyming/alliterating radicals than for words with fully regular radicals (RhyW: Mean $\mathrm{LI}=-0.17, \mathrm{t}(38)=-5.56, p=0.0001)$. The lateralization of activation continued up to the VWFA but decreased, except for the SemW control words, where a semantic radical on the left elicited significantly more activation in the right hemisphere than in the left hemisphere $\left(\right.$ Mean $\left._{\mathrm{LI}}=-0.16, \mathrm{t}(38)=-3.69, p=0.0392\right)$.

To make sure that the differences we observed in posFG between PhoW words with phonetic radicals to the left and to the right were due to phonology and not to orthographic differences between the two types of words, we examined whether the left-right difference was larger for the PhoW words than for the orthographic control SemW words. We ran a $2 * 2$ two-way repeated measures ANOVA for PhoW words and SemW control words to investigate the interaction effect between word type and position of the shared radical in posFG. A significant interaction could provide evidence that the phonology effect is larger than the orthographic effect, and thus that the effect cannot be fully explained by orthographic differences. As it turned out, the ANOVA analysis was borderline $(\mathrm{F}(1,38)=3.843, p=0.057)$, meaning that we have to be cautious in the interpretation. There were two outliers with a score greater than 3 or less than $-3 \mathrm{Z}$-score value. When these persons were left out of the analysis, the results largely remained the same but the interaction effect was significant $(F(1,36)=6.229, p=0.017)$. The figure of the analysis with the outliers removed is shown in Appendix B. 

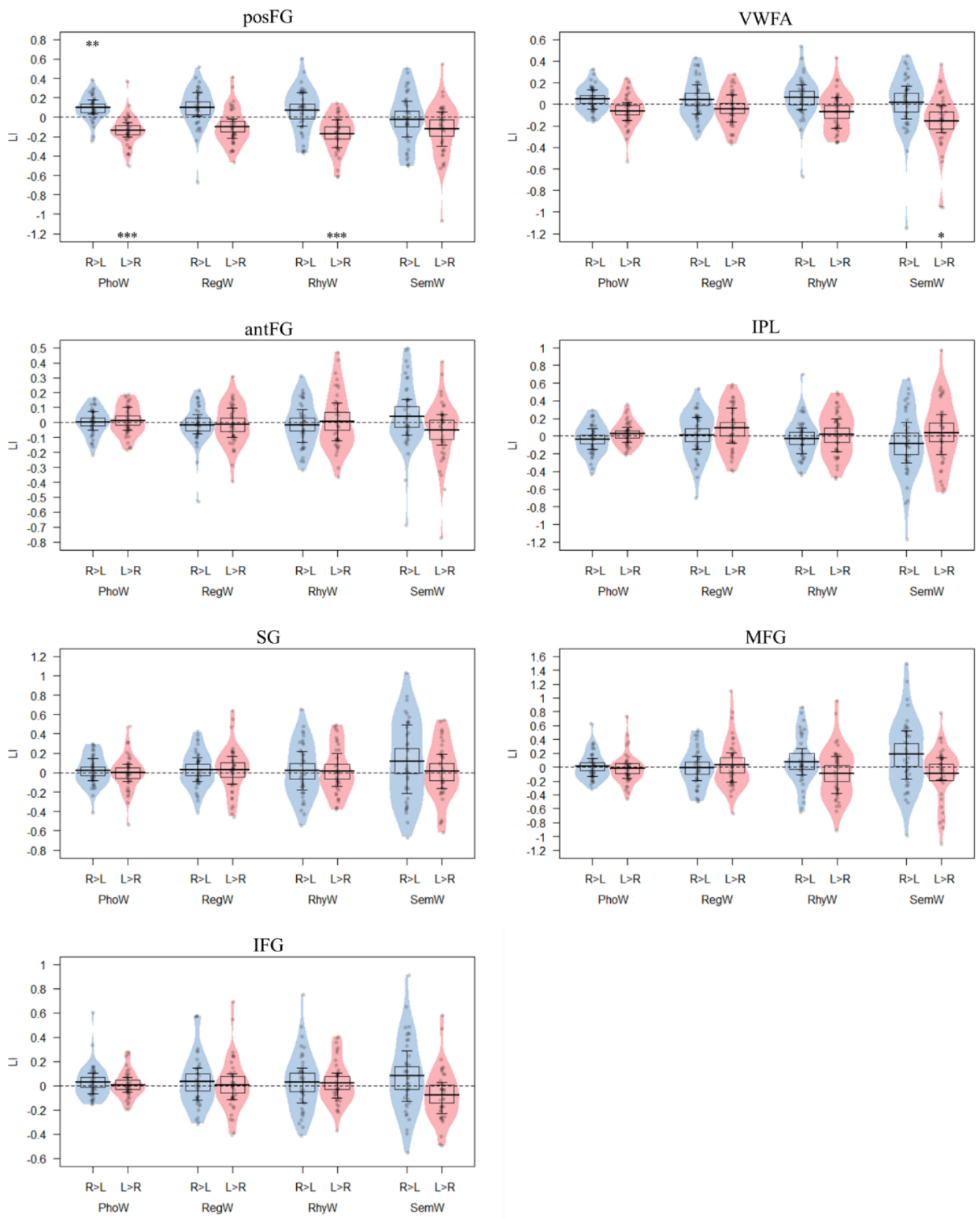

Fig. 5. The distribution of lateralization measures based on the effect size for all types of words and all regions (top 10\% of voxels). In each panel, the y-axis indicates the individual laterality indices $(\mathrm{LI}$, positive values $=$ left lateralization, negative values $=$ right lateralization.), and the $x$-axis indicates the contrasts of interest, specifically, $R>L$ : the 
characters with the critical radical on the right against the characters with the radical on the left; $L>R$ : the characters with the critical radical on the left against the characters with the radical on the right. The boxplots represent the mean LIs and interquartile ranges. Asterisks indicate whether the obtained LI index differs significantly from 0 in a one-sampled t-test (*: $\mathbf{p}<0.05 ; * *: \mathbf{p}<0.01 ; * * *: \mathbf{p}<0.001 ;$ Bonferroni correction for the 7 regions).

The laterality patterns became stronger when the top $20 \%$ of voxels were selected as the fROI, instead of the top 10\%, as can be seen in Fig. 6. In particular, the interaction between position of the radical in PhoW words and SemW words within the posFG was significant both when all data were included (Fig. 6, F $(1,38)=4.993, p=0.031)$ and when the outliers were deleted (Appendix $\mathrm{C}, \mathrm{F}(1,36)=8.282, p=0.007)$. 

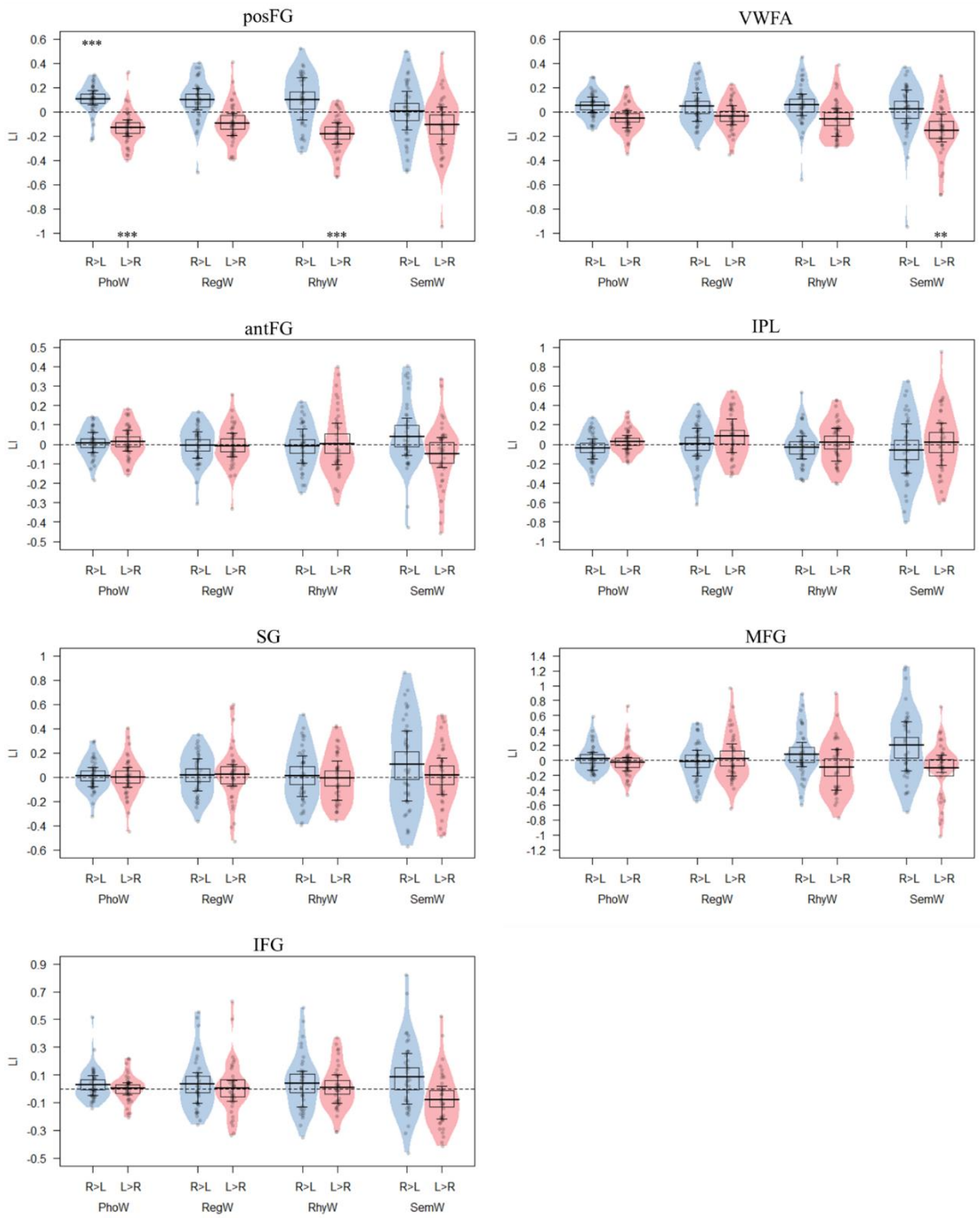

Fig. 6. The distribution of lateralization measures based on the effect size for all types of words and all regions (top $20 \%$ of voxels). In each panel, the y-axis indicates the individual laterality indices $(\mathrm{LI}$, positive values $=$ left lateralization, negative values $=$ right lateralization.), and the $x$-axis indicates the contrasts of interest, specifically, $R>L$ : the 
characters with the critical radical on the right against the characters with the radical on the left; $L>R$ : the characters with the critical radical on the left against the characters with the radical on the right. Asterisks indicate whether the obtained LI index differs significantly from 0 in a one-sampled t-test $(*: p<0.05 ; * *: p<0.01 ; * * *: p<0.001$; Bonferroni correction for the 7 regions).

The second analysis made use of the LI toolbox, which looks at total activation in predefined anatomical regions. For this analysis, different from the first one, participants may not always show enough activation for all contrasts within a predefined ROI. For instance, in posFG the data of one participant was absent for RegW because there were not enough voxels activated on the right side of the masked image (below 20). Participants with missing observations were left out for that particular analysis, as can be seen in the degrees of freedom. As fig. 7 shows, this analysis confirms the clear lateralized activity in the posterior fusiform gyrus, both when activation is calculated on the basis of $\mathrm{R}>\mathrm{L}$ and $\mathrm{L}>\mathrm{R}$ contrasts. Specifically, within posFG the PhoW stimuli with the phonetic radical on the right evoked significant left lateralized activation $\left(\mathrm{Mean}_{\mathrm{LI}}=0.40\right.$, $\mathrm{t}(37)=5.20, p=0.0004)$. Characters with phonetic radicals on the left elicited significantly more right hemisphere activity $\left(\mathrm{Mean}_{\mathrm{LI}}=-0.51, \mathrm{t}(37)=-6.90, p<0.0001\right)$. This was also true for the control SemW words $\left(\right.$ Mean $\left._{L I}=-0.30, \mathrm{t}(37)=-3.98, p=0.0172\right)$. Against our expectations, this analysis failed to show clear lateralized activity in the VWFA. Another interesting observation is that SemW characters elicit more activation in the left anterior fusiform gyrus when the semantic radical was situated on the right $\left(\mathrm{Mean}_{\mathrm{LI}}=0.25, \mathrm{t}(38)=4.07, p=0.0129\right)$. A two-way repeated measures ANOVA indicated that the left-right difference was larger for PhoW words in posFG than for the SemW stimuli $(\mathrm{F}(1,32)=11.069, p=0.002)$. In these analyses there were no outliers with standardized values smaller than -3 or larger than +3 . 

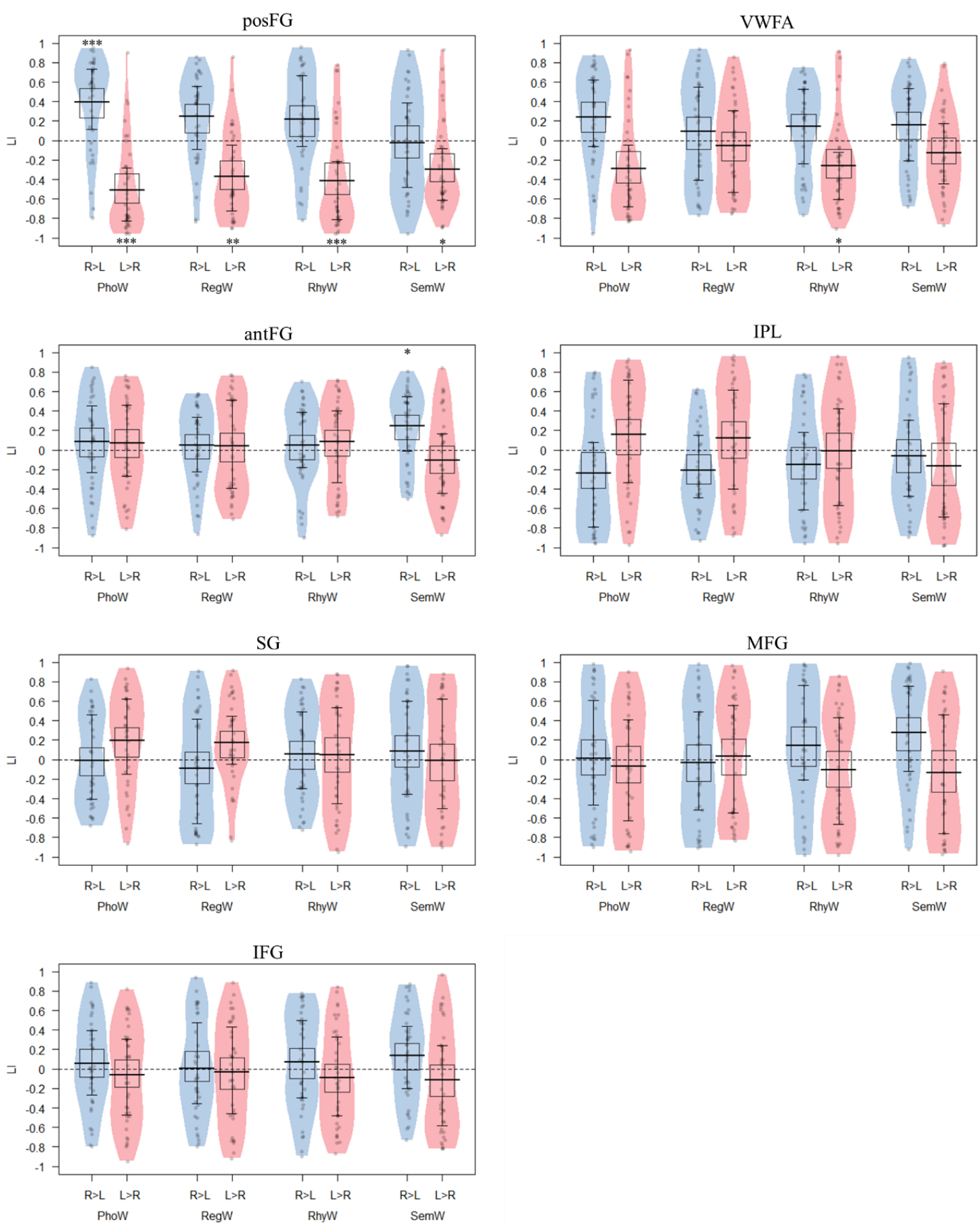

Fig. 7. The distribution of lateralization measures based on the LI toolbox for all types of words and all regions. In each panel, the y-axis indicates the individual laterality indices (LI, 
positive values $=$ left lateralization, negative values $=$ right lateralization. $)$, and the $x$-axis indicates the contrasts of interest, specifically, $R>L$ : the characters with the critical radical on the right against the characters with the radical on the left; $L>R$ : the characters with the critical radical on the left against the characters with the radical on the right. Asterisks indicate whether the obtained LI index differs significantly from 0 in a one-sampled t-test (*: $\mathbf{p}<0.05 ; * *: \mathbf{p}<0.01 ; * * *: \mathbf{p}<0.001$; Bonferroni correction for the 7 regions).

Finally, we investigated the laterality for all word types by using the voxel-based laterality maps (Seghier \& Price, 2011). This analysis (shown Fig. 8, AFNI software, Cox, 1996) confirmed the lateralized activity as a function of phonetic radical position in posFG and extended it to VWFA. There was a similar trend for the SemW control words, but this was much weaker. As suggested by the whole brain analysis (Fig. 4), antFG and MFG were also involved in the lateralized processing of these characters. However, the number of voxels in these areas were very small: there was only 1 significant voxel for the left and the right hemisphere within antFG, and there were only 2 significant voxels for the left and the right hemisphere within the MFG. In fig. 8, we showed the lateralized activity for all types of words when the ROI was limited to the fusiform gyrus. 


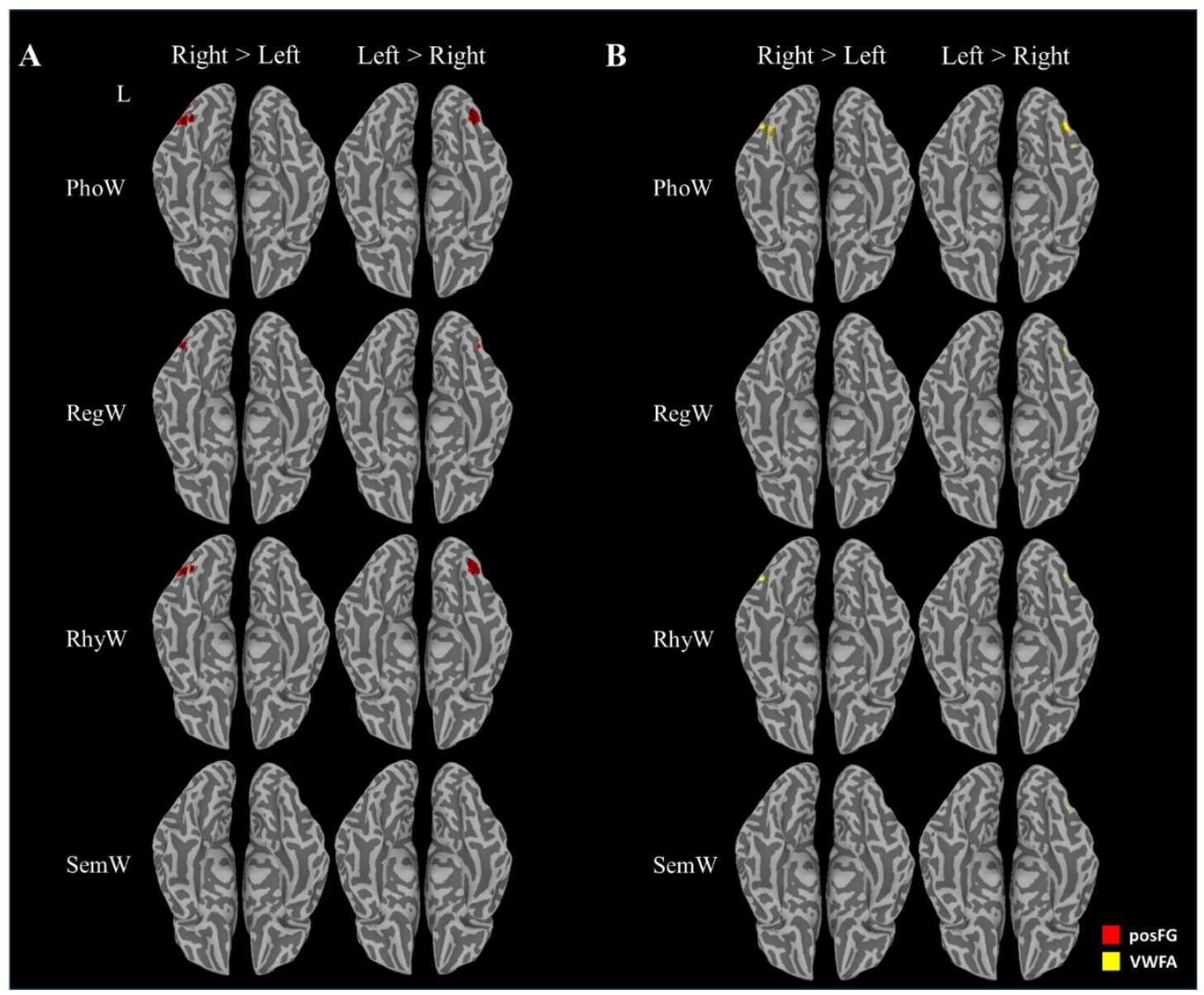

Fig. 8. A: the voxel-based laterality maps for all contrasts at posFG with the inferior view. This figure represents the interaction between the contrast and the hemisphere. Left column shows the regions related to the laterality of characters with the critical radical on the right, and the right column shows the regions related to the laterality of characters with the critical radical on the left. B: the voxel-based laterality maps for all contrasts within the VWFA. Right >Left contrasts the activation for characters with the critical radical on the right with matched characters with the radical on the left; Left > Right contrast the activation for characters with the radical on the left to those with the radical on the right. The ROIs with the color in red is posFG, yellow is VWFA. Significant at $P<0.05$ (voxel-wise, FWEcorrected). L: the left hemisphere. 


\section{Discussion}

In this study we sought to obtain more information about the role of phonology in visual word recognition by examining brain activity in response to Chinese characters containing sub-lexical phonetic information. In particular, we compared characters with a phonetic radical in the left half to characters with a phonetic radical in the right half (respectively Ps and sP characters). These characters have been used before by Hsiao and colleagues to study Chinese word processing (Hsiao et al., 2007; Hsiao \& Liu, 2010). The authors reported that sP characters (with the phonetic radical to the right) elicited left-lateralized processing in an early ERP component (N1, around 180-200 ms post-stimulus), whereas there was no lateralized processing for Ps characters. Source analysis suggested that the difference in $\mathrm{N} 1$ was located in the fusiform gyrus and the inferior occipital cortex (close to the areas VWFA and posFG in Fig. 3).

The findings of Hsiao and colleagues are intriguing because they suggest that Ps and $\mathrm{sP}$ characters may result in processing differences between the left and the right brain half, which we can pick up in an fMRI study. Such hemispheric differences are predicted by the split-fovea hypothesis (Hsiao \& Shillcock, 2005; Ellis \& Brysbaert, 2010; Van der Haegen et al., 2013), according to which the right half of a centrally fixated word is projected to the left hemisphere and the left half to the right hemisphere. This means that the phonetic radical in a sP character is sent initially to the left hemisphere, whereas the phonetic radical in a Ps character is projected initially to the right hemisphere.

To make sure that we were investigating phonological processes, the Chinese characters we used were matched on the critical radical, word frequency, and number of strokes. In addition, we had a control set of ideographs that did not contain phonetic radicals, but had matched semantic radicals on the left or the right. 
A whole brain analysis (Table 2, Fig. 4) confirmed the finding of Hsiao et al. (2007; Hsiao \& Liu, 2010) that phonological information affects processing in the contralateral hemisphere. When participants read sP characters, activity was significantly larger in the posterior fusiform cortex of the left hemisphere, whereas Ps characters evoked more activation in the homologue part of the right hemisphere (Fig. 5-8). The asymmetry was stronger than the asymmetry observed for semantic control stimuli that did not include phonological information, adding evidence to the hypothesis that the effect is phonology-based. The finding that phonetic radicals elicited extra activation in the contralateral occipito-temporal cortex is evidence for the split-fovea hypothesis, according to which there is no region of bilateral information projection at the center of the visual field (Ellis \& Brysbaert, 2010; Van der Haegen et al., 2013).

A further interesting finding is that RhyW characters elicited more activation than RegW characters (Table 2, Fig. 4; see also Zhao et al., 2012). RegW stimuli are words in which the phonetic radical has the same pronunciation as the character; in RhyW stimuli the overlap is only partial (the onset or the rhyme). Behavioral data also show that reading RhyW characters requires more time than reading RegW characters (e.g. Lee et al., 2005). Clearly, the conflict between the phonological information in the phonetic radical and the name of the complete word in RhyW stimuli blocks required extra effort from the participants, even though participants did not have to name the characters.

Unexpectedly, SemW characters with no phonetic radical also modulated activation in the posterior fusiform cortex. This was particularly true for the $\mathrm{sS}$ words with the semantic radical to the right. It has been reported before that activation in the fusiform gyrus is affected by word meaning, but this was particularly true for the medial and anterior portions (Wheatley et al., 2005; Balsamo et al., 2006; Mion et al., 2010; see also Wang et al., 2019, for evidence in logographic 
script). Further research will have to indicate whether the posterior fusiform gyrus is indeed affected by word meaning, or whether the difference in activation could be explained by low-level orthographic factors.

Beyond vOT, SemW characters activated a network of brain regions in line with the predictions of Tan et al. (2005) for Chinese words. As shown in Fig. 2, these authors argued for the involvement of the middle frontal gyrus and the inferior parietal cortex in the processing of words that cannot make use of assembled phonology (remember that SemW words did not contain phonetic radicals). In contrast, the words with phonetic radicals showed enhanced activity in the angular gyrus (but not in IFG), also in line with Tan et al.'s predictions. The extra activity was particularly observed when the information was presented at an infrequent position: phonological information in the left half of the word and semantic information in the right half. Indeed, most Chinese characters have semantic information in the left half and phonological information in the right half. This also seems to be the ideal distribution, given that in the vast majority of people the left hemisphere contains the speech center, so that phonetic radicals have direct connection to the speech center when they are in the right half of the character (Cai et al., 2008; Vingerhoets, 2019). Unfortunately, the whole-brain findings were not fully backed up by more detailed ROI analyses. In these analyses we calculated three different laterality indexes for seven brain areas predefined on the basis of a literature review: bilateral VWFA, posFG, antFG, IPL, SG, MFG, and IFG. These areas cover the word reading network (Dehaene, 2009; Tan et al., 2005; see Fig. 1). Only in posFG did LI analysis provide consistent evidence for left lateralization in the processing of $\mathrm{sP}$ words and right lateralization in the processing of Ps words (Fig. 5-8). The other ROIs showed interesting trends in line with the whole brain analysis, but the evidence was not consistent enough across LI measures to make strong claims on the basis of the present data alone. 
At first sight it is surprising that posFG showed the largest difference between characters with left and right phonetic radicals. After all, this is an area in the visual cortex, which is more responsible for visual feature extraction of visual word recognition (Lerma-Usabiaga et al., 2018) but not typically associated with phonological processing (remember that the lateralization was stronger for PhoW stimuli than for SemW stimuli). However, this finding is in line with Price and Devlin's (2011) model of visual word recognition, which assumes highly interactive processes between the anterior language areas in the frontal cortex and the posterior language areas in the occipitotemporal cortex (for empirical evidence related to the model, see Barca et al., 2011; Chu \& Meltzer, 2019; Kerry et al., 2019; Pammer et al., 2004; Selpien et al., 2015; Stevens et al., 2017; Strother et al., 2016, 2017; Woodhead et al., 2013, 2014). Woodhead et al. (2014) is a particularly relevant study. They used MEG to compare the processing of short visual English words vs. the processing of matched stimuli that did not include verbal information (false fonts). Three bilateral ROIs were defined: (1) OCC in the occipital cortex (centered on $\pm 15-952$, close to Strother et al.'s occipital word form area), (2) vOT (centered on $\pm 44-58-15$, coinciding with VWFA), and (3) IFG (centered on \pm 4828 0). Using dynamic causal modelling, the authors found that in the first 300 ms for words there was extra information transfer from left OCC to left vOT and from left OCC to left IFG. In addition, there was increased communication from left IFG to left vOT, in line with Price and Devlin's (2011) interactive model. Lerma-Usabiaga et al. (2018) also reported evidence for direct connections between the posterior area they identified and the intraparietal sulcus through the vertical occipital fasciculus.

On the basis of Woodhead et al. (2014), we had hoped to find lateralization in VWFA and IFG for PhoW stimuli. There were some trends in line with the expectations in VWFA but not in IFG (Fig. 5-8). In hindsight, we may have expected too much, given that fMRI does not have the temporal 
resolution of MEG and given that the words in the various conditions were matched very carefully, so that the only difference between them was the position of the phonetic radical. Also the middle frontal gyrus seems to be more important in Chinese character recognition than IFG (Tan et al., 2005; Fig. 2). Still, the finding that posFG responds strongly to phonetic information strongly suggests the existence of feedback from higher language regions to posFG (most likely from IFG and/or MFG).

In summary, our data show that visual information from centrally presented Chinese characters is split in the fovea and sent to the contralateral visual cortex, from which phonological information can be extracted rapidly if the character contains a phonetic radical. The phonological information helps word processing by means of top-down feedback. Extra activation, suggestive of more effortful processing, is observed when the phonetic radical is situated in the left half of the character and therefore initially sent to the visual cortex in the right hemisphere that is less specialized for language processing. 


\section{Acknowledgements}

We would like to thank Qing Cai for her help with the stimulus selection and Helena Verhelst for help with the collection of data. This work was supported by a grant awarded by the Ghent University (CCN-kas D/01329/01, Belgium) to prof. Marc Brysbaert and a studentship to Xiaodong Liu (Oversea Study Program of Guangzhou Elite Project, JY201717, China).

\section{Open practices}

Anonymized data, analysis code, materials and the fMRI paradigms are available at https://osf.io/5k2wn/. We report how we determined our sample size, all data exclusions (if any), all inclusion/exclusion criteria, whether inclusion/exclusion criteria were established prior to data analysis, all manipulations, and all measures in the study. No part of the study procedures and analyses were pre-registered prior to the research being conducted.

\section{CRediT authorship contribution statement}

Xiaodong Liu: Investigation, Formal analysis, Data Curation, Writing - original draft, Writing review \& editing. Luc Vermeylen: Investigation, Writing - Original Draft. David Wisniewski: Formal analysis, Writing - Original Draft. Marc Brysbaert: Conceptualization, Methodology, Writing - original draft; Writing - review \& editing, Supervision, Funding acquisition.

\section{Declaration of Competing Interest}

The authors declare that they have no financial disclosures or potential conflicts of interests. 


\section{References}

Alan, K., Joël, P., \& Stéphanie, D. (2002). Parafoveal-on-foveal interactions in word recognition. The Quarterly Journal of Experimental Psychology Section A, 55(4), 1307-1337. https://doi.org/10.1080/02724980244000071

Alvarez, T. A., \& Fiez, J. A. (2018). Current Perspectives on the Cerebellum and Reading Development. Neuroscience and Biobehavioral Reviews, 92, 55-66. https://doi.org/10.1016/j.neubiorev.2018.05.006

Balsamo, L. M., Xu, B., \& Gaillard, W. D. (2006). Language lateralization and the role of the fusiform gyrus in semantic processing in young children. NeuroImage, 31(3), 1306-1314. https://doi.org/10.1016/j.neuroimage.2006.01.027

Barca, L., Cornelissen, P., Simpson, M., Urooj, U., Woods, W., \& Ellis, A. W. (2011). The neural basis of the right visual field advantage in reading: An MEG analysis using virtual $\begin{array}{lllll}\text { electrodes. } & \text { Brain }\end{array}$ https://doi.org/10.1016/j.bandl.2010.09.003

Bolger, D. J., Perfetti, C. A., \& Schneider, W. (2005). Cross-cultural effect on the brain revisited: universal structures plus writing system variation. Human Brain Mapping, 25(1), 92-104. https://doi.org/10.1002/hbm.20124

Borowsky, R., Cummine, J., Owen, W. J., Friesen, C. K., Shih, F., \& Sarty, G. E. (2006). FMRI of Ventral and Dorsal Processing Streams in Basic Reading Processes: Insular Sensitivity to Phonology. Brain Topography, 18(4), 233-239. https://doi.org/10.1007/s10548-006$0001-2$ 
Brysbaert, M. (2004). The importance of interhemispheric transfer for foveal vision: A factor that has been overlooked in theories of visual word recognition and object perception. Brain and Language, 88(3), 259-267. https://doi.org/10.1016/s0093-934x(03)00279-7

Cai, Q., Paulignan, Y., Brysbaert, M., Ibarrola, D., \& Nazir, T. A. (2010). The Left Ventral Occipito-Temporal Response to Words Depends on Language Lateralization but Not on Visual Familiarity. Cerebral Cortex, 20(5), 1153-1163. https://doi.org/10.1093/cercor/bhp175

Cai, Q., Lavidor, M., Brysbaert, M., Paulignan, Y., \& Nazir, T. A. (2008). Cerebral Lateralization of Frontal Lobe Language Processes and Lateralization of the Posterior Visual Word Processing System. Journal of Cognitive Neuroscience, 20(4), 672-681. https://doi.org/10.1162/jocn.2008.20043

Carreiras, M., Armstrong, B. C., Perea, M., \& Frost, R. (2014). The what, when, where, and how of visual word recognition. Trends in Cognitive Sciences, 18(2), 90-98. https://doi.org/10.1016/j.tics.2013.11.005

Chu, R. K., \& Meltzer, J. A. (2019). Interhemispheric connectivity during lateralized lexical decision. Human Brain Mapping, 40(3), 818-832. https://doi.org/10.1002/hbm.24414

Cocquyt, E. M., Coffé, C., van Mierlo, P., Duyck, W., Mariën, P., Szmalec, A., ... De Letter, M. (2019). The involvement of subcortical grey matter in verbal semantic comprehension: A systematic review and meta-analysis of fMRI and PET studies. Journal of Neurolinguistics, 51, 278-296. https://doi.org/10.1016/j.jneuroling.2019.04.001 
Cohen, L., Lehéricy, S., Chochon, F., Lemer, C., Rivaud, S., \& Dehaene, S. (2002). Languagespecific tuning of visual cortex? Functional properties of the Visual Word Form Area. Brain, 125(5), 1054-1069. https://doi.org/10.1093/brain/awf094

Cox, R. W. (1996). AFNI: Software for Analysis and Visualization of Functional Magnetic Resonance Neuroimages. Computers and Biomedical Research, 29(3), 162-173. https://doi.org/10.1006/cbmr.1996.0014

Dehaene. S. (2009). Reading in the brain: the science and evolution of a human invention. New York : Viking.

Ellis, A. W., \& Brysbaert, M. (2010). Split fovea theory and the role of the two cerebral hemispheres in reading: A review of the evidence. Neuropsychologia, 48(2), 353-365. https://doi.org/10.1016/j.neuropsychologia.2009.08.021

Fedorenko, E., Hsieh, P.-J., Nieto-Castañón, A., Whitfield-Gabrieli, S., \& Kanwisher, N. (2010). New Method for fMRI Investigations of Language: Defining ROIs Functionally in Individual Subjects. Journal of Neurophysiology, 104(2), 1177-1194. https://doi.org/10.1152/jn.00032.2010

Friston, K. J., Jezzard, P., \& Turner, R. (1994). Analysis of functional MRI time-series. Human Brain Mapping, 1(2), 153-171. https://doi.org/10.1002/hbm.460010207

Hirshorn, E. A., Wrencher, A., Durisko, C., Moore, M. W., \& Fiez, J. A. (2016). Fusiform Gyrus Laterality in Writing Systems with Different Mapping Principles: An Artificial Orthography Training Study. Journal of Cognitive Neuroscience, 28(6), 882-894. https://doi.org/10.1162/jocn_a_00940 
Hsiao, J. H., \& Liu, T. (2010). Position of phonetic components may influence how written words are processed in the brain: Evidence from Chinese phonetic compound pronunciation. Cognitive, Affective, \& Behavioral Neuroscience, 10(4), 552-559. https://doi.org/10.3758/cabn.10.4.552

Hsiao, J. H., \& Shillcock, R. (2005). Foveal splitting causes differential processing of Chinese orthography in the male and female brain. Cognitive Brain Research, 25(2), 531-536. https://doi.org/10.1016/j.cogbrainres.2005.08.005

Hsiao, J. H., \& Shillcock, R. (2006). Analysis of a Chinese Phonetic Compound Database: Implications for Orthographic Processing. Journal of Psycholinguistic Research, 35(5), 405-426. https://doi.org/10.1007/s10936-006-9022-y

Hsiao, J. H., Shillcock, R., \& Lee, C. (2007). Neural correlates of foveal splitting in reading: Evidence from an ERP study of Chinese character recognition. Neuropsychologia, 45(6), 1280-1292. https://doi.org/10.1016/j.neuropsychologia.2006.10.001

Hue, C.-W. (1992). Recognition processes in character naming. In H. C. Chen \& O. J. L. Tzeng (Eds.), Language processing in Chinese (pp. 93-107). Amsterdam: North-Holland.

Jobard, G., Crivello, F., \& Tzourio-Mazoyer, N. (2003). Evaluation of the dual route theory of reading: a metanalysis of 35 neuroimaging studies. NeuroImage, 20(2), 693-712. https://doi.org/10.1016/s1053-8119(03)00343-4

Jun, D. (2010). Jun Da: Chinese text computing. Retrieved from lingua.mtsu.edu website: http://lingua.mtsu.edu/chinese-computing. September 2010. 
Kawabata Duncan, K. J., Twomey, T., Parker Jones, O., Seghier, M. L., Haji, T., Sakai, K., ... Devlin, J. T. (2014). Inter- and Intrahemispheric Connectivity Differences When Reading Japanese Kanji and Hiragana. Cerebral Cortex, 24(6), 1601-1608. https://doi.org/10.1093/cercor/bht015

Kerry, S. J., Aguilar, O. M., Penny, W., Crinion, J. T., Leff, A. P., \& Woodhead, Z. V. J. (2019). How Does iReadMore Therapy Change the Reading Network of Patients with Central Alexia? The Journal of Neuroscience, 39(29), 5719-5727. https://doi.org/10.1523/jneurosci.1426-18.2019

Kriegeskorte, N., Simmons, W. K., Bellgowan, P. S. F., \& Baker, C. I. (2009). Circular analysis in systems neuroscience: the dangers of double dipping. Nature Neuroscience, 12(5), 535540. https://doi.org/10.1038/nn.2303

Lee, C.-Y., Tsai, J.-L., Su, E. C.-I., Tzeng, O. J. L., \& Hung, D. L. (2005). Consistency, Regularity, and Frequency Effects in Naming Chinese Characters. Language and Linguistics, 6(1), 75107.

Lerma-Usabiaga, G., Carreiras, M., \& Paz-Alonso, P. M. (2018). Converging evidence for functional and structural segregation within the left ventral occipitotemporal cortex in reading. Proceedings of the National Academy of Sciences, 115(42), E9981-E9990.

Li, Y., \& Kang, J. S. (1993). Analysis of phonetics of the ideophonetic characters in Modern Chinese. In Y. Chen (Ed.), Information analysis of usage of characters in modern Chinese (pp. 84-98). Shanghai: Shanghai Education Publisher (in Chinese).

Mahowald, K., \& Fedorenko, E. (2016). Reliable individual-level neural markers of high-level language processing: A necessary precursor for relating neural variability to behavioral and 


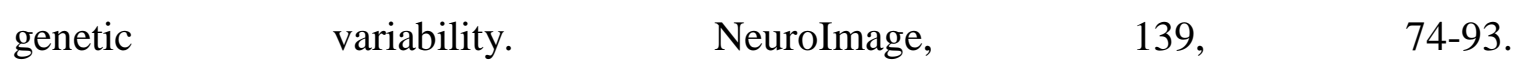
https://doi.org/10.1016/j.neuroimage.2016.05.073

Mariën, P., Paghera, B., De Deyn, P. P., \& Vignolo, L. A. (2004). Adult Crossed Aphasia in Dextrals Revisited. Cortex, 40(1), 41-74. https://doi.org/10.1016/s0010-9452(08)70920-1

Mion, M., Patterson, K., Acosta-Cabronero, J., Pengas, G., Izquierdo-Garcia, D., Hong, Y. T., ... Nestor, P. J. (2010). What the left and right anterior fusiform gyri tell us about semantic memory. Brain, 133(11), 3256-3268. https://doi.org/10.1093/brain/awq272

Oldfield, R. C. (1971). The assessment and analysis of handedness: The Edinburgh inventory. Neuropsychologia, 9(1), 97-113. https://doi.org/10.1016/0028-3932(71)90067-4

Pammer, K., Hansen, P. C., Kringelbach, M. L., Holliday, I., Barnes, G., Hillebrand, A., ... Cornelissen, P. L. (2004). Visual word recognition: the first half second. NeuroImage, 22(4), 1819-1825. https://doi.org/10.1016/j.neuroimage.2004.05.004

Peirce, J., Gray, J. R., Simpson, S., MacAskill, M., Höchenberger, R., Sogo, H., .. Lindeløv, J. K. (2019). PsychoPy2: Experiments in behavior made easy. Behavior Research Methods, 51(1), 195-203. https://doi.org/10.3758/s13428-018-01193-y

Price, C. J., \& Devlin, J. T. (2011). The Interactive Account of ventral occipitotemporal contributions to reading. Trends in Cognitive Sciences, 15(6), 246-253. https://doi.org/10.1016/j.tics.2011.04.001

Schroyens, W., Vitu, F., Brysbaert, M., \& d'Ydewalle, G. (1999).Visual attention and eyemovement control during reading: The case of parafoveal processing. Quarterly Journal of Experimental Psychology, 52A, 1021-1046. 
Seghier, M. L., \& Price, C. J. (2011). Explaining Left Lateralization for Words in the Ventral Occipitotemporal Cortex. Journal of Neuroscience, 31(41), 14745-14753. https://doi.org/10.1523/jneurosci.2238-11.2011

Selpien, H., Siebert, C., Genc, E., Beste, C., Faustmann, P. M., Güntürkün, O., \& Ocklenburg, S. (2015). Left dominance for language perception starts in the extrastriate cortex: An ERP and sLORETA study. Behavioural Brain Research, 291, 325-333. https://doi.org/10.1016/j.bbr.2015.05.050

Stevens, W. D., Kravitz, D. J., Peng, C. S., Tessler, M. H., \& Martin, A. (2017). Privileged Functional Connectivity between the Visual Word Form Area and the Language System. The Journal of Neuroscience, 37(21), 5288-5297. https://doi.org/10.1523/jneurosci.013817.2017

Strother, L., Coros, A. M., \& Vilis, T. (2016). Visual Cortical Representation of Whole Words and Hemifield-split Word Parts. Journal of Cognitive Neuroscience, 28(2), 252-260. https://doi.org/10.1162/jocn_a_00900

Strother, L., Zhou, Z., Coros, A. K., \& Vilis, T. (2017). An fMRI study of visual hemifield integration and cerebral lateralization. Neuropsychologia, 100, 35-43. https://doi.org/10.1016/j.neuropsychologia.2017.04.003

Tan, L. H., Laird, A. R., Li, K., \& Fox, P. T. (2005). Neuroanatomical correlates of phonological processing of Chinese characters and alphabetic words: A meta-analysis. Human Brain Mapping, 25(1), 83-91. https://doi.org/10.1002/hbm.20134 
Taylor, J. S. H., Rastle, K., \& Davis, M. H. (2013). Can cognitive models explain brain activation during word and pseudoword reading? A meta-analysis of 36 neuroimaging studies. Psychological Bulletin, 139(4), 766-791. https://doi.org/10.1037/a0030266

Twomey, T., Kawabata Duncan, K. J., Price, C. J., \& Devlin, J. T. (2011). Top-down modulation of ventral occipito-temporal responses during visual word recognition. NeuroImage, 55(3), 1242-1251. https://doi.org/10.1016/j.neuroimage.2011.01.001

Tzourio-Mazoyer, N., Landeau, B., Papathanassiou, D., Crivello, F., Etard, O., Delcroix, N., ... Joliot, M. (2002). Automated Anatomical Labeling of Activations in SPM Using a Macroscopic Anatomical Parcellation of the MNI MRI Single-Subject Brain. NeuroImage, 15(1), 273-289. https://doi.org/10.1006/nimg.2001.0978

Van der Haegen, L., Cai, Q., \& Brysbaert, M. (2012). Colateralization of Broca's area and the visual word form area in left-handers: fMRI evidence. Brain and Language, 122(3), 171178. https://doi.org/10.1016/j.bandl.2011.11.004

Van der Haegen, L., Cai, Q., Stevens, M. A., \& Brysbaert, M. (2013). Interhemispheric Communication Influences Reading Behavior. Journal of Cognitive Neuroscience, 25(9), 1442-1452. https://doi.org/10.1162/jocn_a_00412

Van Heuven, W. J. B., Mandera, P., Keuleers, E., \& Brysbaert, M. (2014). Subtlex-UK: A New and Improved Word Frequency Database for British English. Quarterly Journal of Experimental Psychology, $\quad$ 67(6), $\quad$ 1176-1190. https://doi.org/10.1080/17470218.2013.850521

Vingerhoets, G. (2019). Phenotypes in hemispheric functional segregation? Perspectives and challenges. Physics of Life Reviews, 30, 1-18. https://doi.org/10.1016/j.plrev.2019.06.002 
Wandell, B. A., \& Le, R. K. (2017). Diagnosing the Neural Circuitry of Reading. Neuron, 96(2), 298-311. https://doi.org/10.1016/j.neuron.2017.08.007

Wang, J., Deng, Y., \& Booth, J. R. (2019). Automatic semantic influence on early visual word recognition in the ventral occipito-temporal cortex. Neuropsychologia, 133, 107188. https://doi.org/10.1016/j.neuropsychologia.2019.107188

Wang, Y. (1996). Relations between the sides of linguistic cerebral dominance and manuality in Chinese aphasics. Chinese Medical Journal, 109(7), 572-575.

Wheatley, T., Weisberg, J., Beauchamp, M. S., \& Martin, A. (2005). Automatic Priming of Semantically Related Words Reduces Activity in the Fusiform Gyrus. Journal of Cognitive Neuroscience, 17(12), 1871-1885. https://doi.org/10.1162/089892905775008689

Wilke, M., \& Lidzba, K. (2007). LI-tool: A new toolbox to assess lateralization in functional MRdata. Journal of Neuroscience Methods, 163(1), 128-136. https://doi.org/10.1016/j.jneumeth.2007.01.026

Wilke, M., \& Schmithorst, V. J. (2006). A combined bootstrap/histogram analysis approach for computing a lateralization index from neuroimaging data. NeuroImage, 33(2), 522-530. https://doi.org/10.1016/j.neuroimage.2006.07.010

Woodhead, Z. V. J., Barnes, G. R., Penny, W., Moran, R., Teki, S., Price, C. J., \& Leff, A. P. (2014). Reading Front to Back: MEG Evidence for Early Feedback Effects During Word Recognition. Cerebral Cortex, 24(3), 817-825. https://doi.org/10.1093/cercor/bhs365 
Woodhead, Z. V. J., Penny, W., Barnes, G. R., Crewes, H., Wise, R. J. S., Price, C. J., \& Leff, A. P. (2013). Reading therapy strengthens top-down connectivity in patients with pure alexia. Brain, 136(8), 2579-2591. https://doi.org/10.1093/brain/awt186

Wu, C.-Y., Ho, M.-H. R., \& Chen, S.-H. A. (2012). A meta-analysis of fMRI studies on Chinese orthographic, phonological, and semantic processing. NeuroImage, 63(1), 381-391. https://doi.org/10.1016/j.neuroimage.2012.06.047

Xia, M., Wang, J., \& He, Y. (2013). BrainNet Viewer: A Network Visualization Tool for Human Brain Connectomics. PLoS ONE, 8(7), e68910. https://doi.org/10.1371/journal.pone.0068910

Zhao, J., Li, Q.-L., Wang, J.-J., Yang, Y., Deng, Y., \& Bi, H.-Y. (2012). Neural basis of phonological processing in second language reading: An fMRI study of Chinese regularity effect. NeuroImage, 60(1), 419-425. https://doi.org/10.1016/j.neuroimage.2011.12.074

Zhao, L., Chen, C., Shao, L., Wang, Y., Xiao, X., Chen, C., ... Xue, G. (2017). Orthographic and Phonological Representations in the Fusiform Cortex. Cerebral Cortex, 27(11), 5197-5210. https://doi.org/10.1093/cercor/bhw300

Zhao, R., Fan, R., Liu, M., Wang, X., \& Yang, J. (2017). Rethinking the function of brain regions for reading Chinese characters in a meta-analysis of fMRI studies. Journal of Neurolinguistics, 44, 120-133. https://doi.org/10.1016/j.jneuroling.2017.04.001

Zhu, L., Nie, Y., Chang, C., Gao, J.-H., \& Niu, Z. (2014). Different patterns and development characteristics of processing written logographic characters and alphabetic words: An ALE meta-analysis. Human Brain $\quad$ Mapping, 35(6), 2607-2618. https://doi.org/10.1002/hbm.22354 
